\title{
Aspiration Via Congenital Broncho-esophageal Fistula After Lobectomy
}

\author{
Xue-Fei Hu MD, Chang Chen MD, Liang Duan MD, Yi Zhang MD, and Wen Gao MD
}

\begin{abstract}
Aspiration via a congenital broncho-esophageal fistula in an adult thoracotomy patient has not been previously reported. Repeated aspiration and subsequent respiratory failure if the fistula is not recognized could be life-threatening in these postoperative patients. We describe one such critical case, in which a broncho-esophageal fistula was discovered weeks after aspiration and the onset of respiratory failure after left lower lobectomy. This unusual case suggests that repeated localized pulmonary infections can indicate a broncho-esophageal fistula and that further investigations should be performed, including detailed history. Key words: bronchial fistula; bronchiectasis; esophageal fistula; surgery. [Respir Care 2011;56(8):1195-1197. (c) 2011 Daedalus Enterprises]
\end{abstract}

\section{Introduction}

Aspiration is one of the most common reasons for postthoracotomy respiratory failure. The risk factors for postoperative aspiration are older age, oropharyngeal dysfunction, and careless ingestion. Congenital bronchoesophageal fistula as the cause of aspiration and resultant respiratory failure immediately after thoracotomy has been largely undocumented and is difficult to manage. We report a patient who developed unexplained gastrointestinal flatulence during the early course of mechanical ventilation, and in whom multiple bronchoscopies failed to reveal endobronchial abnormalities.

\section{Case Report}

A 75-year-old man, $165 \mathrm{~cm}$ tall, and only $40 \mathrm{~kg}$, presented with bloody sputum for one week. Chest radiograph at the local hospital suggested a mass and prominent bronchiectasis in the left lower lobe. His medical history in-

The authors are affiliated with the Department of Thoracic Surgery, Shanghai Pulmonary Hospital, Tongji University School of Medicine, Shanghai, China.

The authors have disclosed no conflicts of interest.

Correspondence: Chang Chen MD, Department of Thoracic Surgery, Tongji University-Affiliated Shanghai Pulmonary Hospital, Zhengmin Road 507, Shanghai 200433, China. E-mail: changchenc@hotmail.com.

DOI: $10.4187 /$ respcare.01116 cluded recurrent pulmonary infections and productive cough for more than 40 years. He declined pulmonary resection because of worries about the operative risk.

On physical examination he was afebrile and had stable vital signs, but there were prominent rales and rhonchi in his left lower chest. Further workup found completely normal blood count, chemistry lab values, and arterial blood gas analysis. Bronchiectasis of the left lower lobe was prominent on both chest radiograph and helical computed tomography. The pulmonary mass in the superior segment of the left lower lobe was highly suggestive of malignancy (Fig. 1). Bronchoscopy showed a neoplasm in the superior segment of the left lower lobe, which biopsy verified to be adenocarcinoma. Endobronchial mucosa at the remaining sites was otherwise normal. After a series of examinations excluded remote metastasis, he underwent left lower lobectomy via thoracotomy. The operation was unremarkable, but there were intense pulmonary adhesions from his long history of recurrent pulmonary infections. Systemic lymph node dissection was performed in the routine way.

On the first postoperative morning, he had cough after his first sip of liquid. Sputum retention became symptomatic on the next day, his arterial oxygen saturation dropped to near $90 \%$, and multiple bedside bronchoscopies were conducted for sputum clearance. On the following 2 days he developed severe bilateral pulmonary infections and respiratory failure, so tracheotomy was performed and mechanical ventilation support was provided. Routine laboratory examination of the chest drainage suggested transudate. There were no signs of pleural infection. 


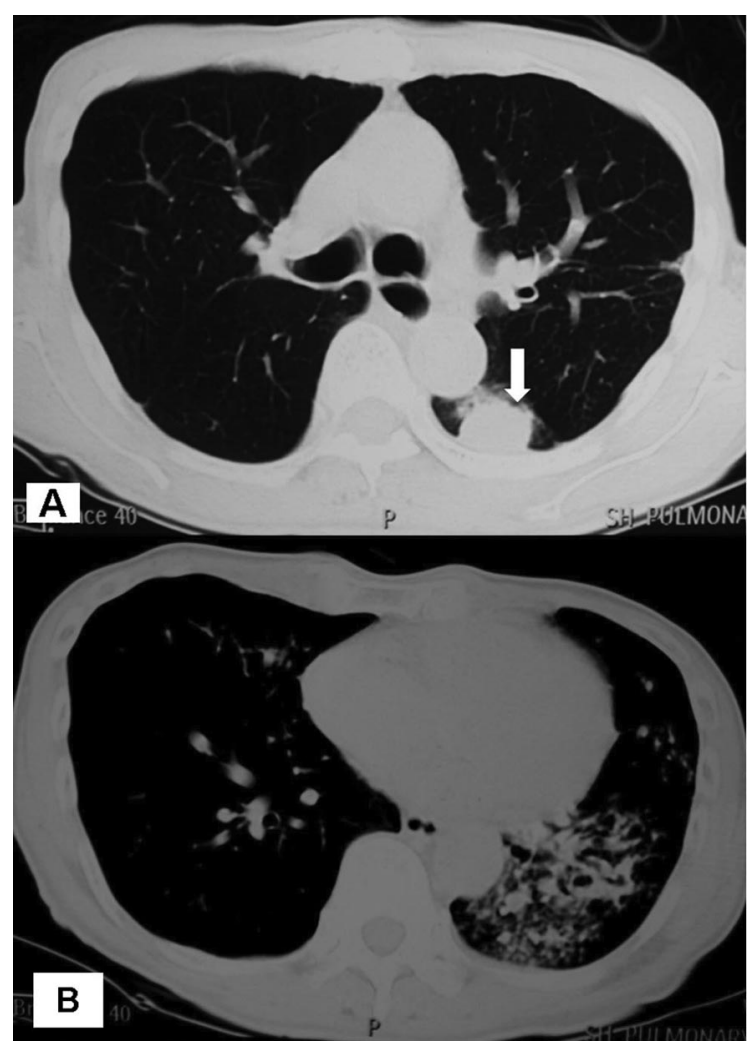

Fig. 1. A and B: Computed tomogram shows prominent bronchiectasis of the left lower lobe and a pulmonary mass (arrow) that is highly suggestive of lung cancer.

The appearance of mild gastrointestinal flatulence since the start of mechanical ventilation raised the suspicion of broncho-esophageal fistula. More than 5 bronchoscopies for mucosa examination and sputum clearance revealed no bronchial defects or dehiscence of the left lower bronchial stump, so the gastric flatulence was reasoned to be the result of air leaking backward into the oropharynx and then being swallowed, and gastric feeding was restored. However, on the 20th postoperative day an incidental withdrawal of the gastric tube caused minor leakage of food into the airway. Immediate bronchoscopy to search for the origin of the leakage revealed a fistula, $3 \mathrm{~mm}$ in diameter, $2 \mathrm{~cm}$ distal to the carina, in a membranous part of the left main bronchus (Fig. 2). Computed tomogram showed a thin septum between the esophagus and the bronchi (Fig. 3). Repeat questioning revealed frequent postprandial coughing in his history. Since he was seriously ill at this time we did not consider surgical repair of the fistula. Instead, we placed a second esophageal gastric tube around the hole for continuous suctioning at $15 \mathrm{~cm} \mathrm{H}_{2} \mathrm{O}$, and this eliminated the gastrointestinal flatulence.

He then gradually recovered from the pulmonary infection and respiratory failure. Pleural infection, shown by a $>50 \%$ neutrocyte component in the purulent chest effusion, was diagnosed on the 25 th postoperative day. Bron-

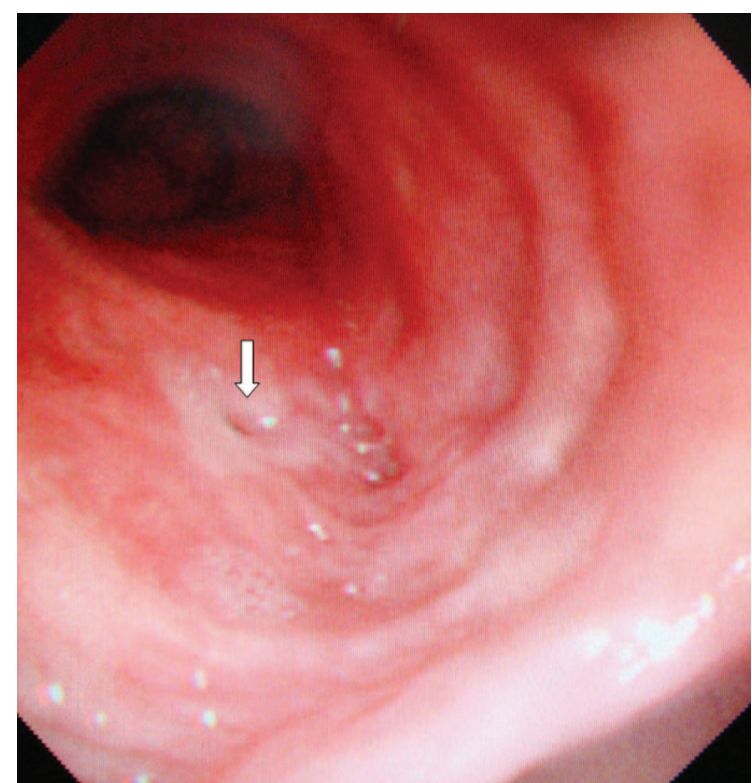

Fig. 2. Bronchoscopy shows a 3-mm broncho-esophageal fistula (arrow) in a membranous part of the left main bronchus. The peripheral pale coloration and probable mucosal edema was obvious only after weeks of esophageal suctioning.

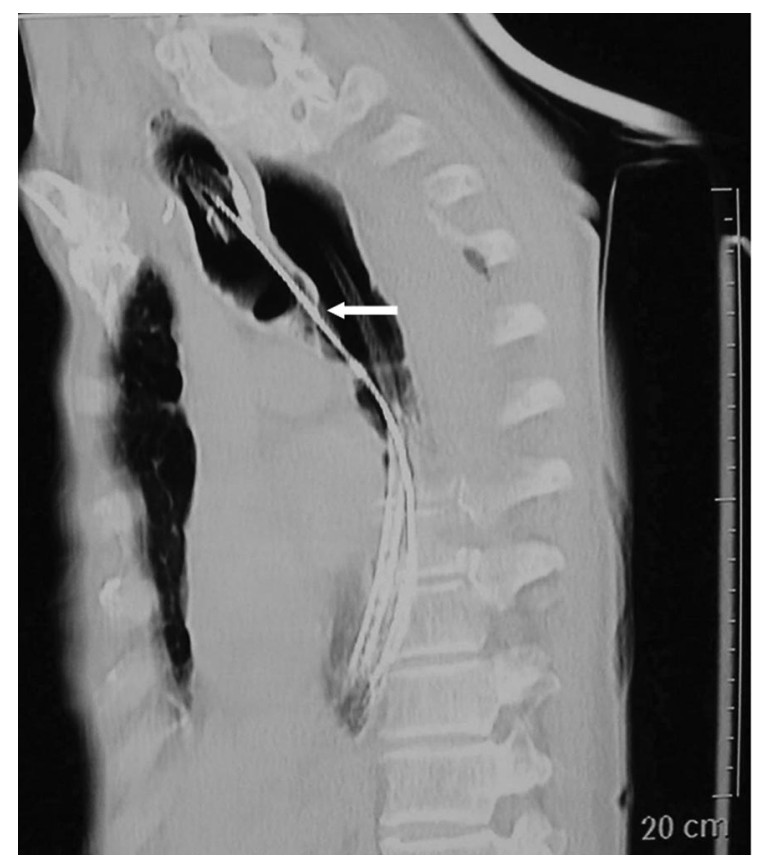

Fig. 3. Sagittal computed tomogram shows a thin septum between the esophagus and the left main bronchus. A guide wire (arrow) was inserted through the fistula.

chial stump dehiscence was visualized a week later, but then he spontaneously healed after the pulmonary infection and empyema were controlled. However, he remained emaciated and weak, so we did not attempt to repair the fistula. After the pulmonary infections were controlled we 
placed a covered endobronchial metal stent in the left main bronchus, then restarted an oral diet. The chest tube was later removed after plural irrigation, and he was discharged on the 107th postoperative day. Final pathology showed a stage Ib (T2NOM0) peripheral adenocarcinoma and bronchiectasis of the left lower lobe.

\section{Discussion}

Whether the broncho-esophageal fistula in our patient was congenital or acquired was the subject of discussion. Generally, diagnosis of a congenital fistula in an adult requires that 3 criteria be met: no surrounding inflammation or malignancy; absence of adherent lymph nodes; and presence of normal mucosa. ${ }^{1}$ Accordingly, the congenital nature of this broncho-esophageal fistula was supported by 5 pieces of evidence. First, he developed aspiration on the first postoperative day, which was also 3 weeks earlier than the appearance of empyema. Had the aspiration been caused by an operative injury, pleural infection would have been the first laboratory finding. Second, there was no mucosal edema around the fistula on early bedside bronchoscopies; the pale color of the peripheral mucus in Figure 2 appeared 4 weeks after intra-esophageal suctioning. Third, there was no regional lymph node metastasis or inflammation. Fourth, later recall of his history revealed casual coughing after drinking, suggesting the possibility of a minor communication between the esophagus and the airway. Broncho-esophageal fistula was also consistent with the history of recurrent pulmonary infection and bronchiectasis in the left lower lobe. Finally, in our succeeding cases of lymphadenectomy, observations excluded the possibility of an incidental cauteric burn synchronically on the abutting esophagus and left main bronchi at a location deep inside the aorta-pulmonary window. Therefore, the present case was a type II congenital broncho-esophageal fistula, according to the Braimbridge and Keith classification. $^{2}$

Preoperative recognition of the fistula may be difficult, ${ }^{3,4}$ although most patients would present the typical symptom of coughing bouts during ingestion. Repeated pulmonary aspiration and infection are usually highly suggestive of broncho-esophageal fistula, which further history inquiry and barium esophagography may reveal. ${ }^{5-7}$

Fistulectomy or esophagectomy is the best treatment for broncho-esophageal fistula. ${ }^{8}$ Covered endobronchial stent provides only palliation for restoring oral feeding and is indicated only in patients who are poor candidates for surgery, as was our patient.

\section{REFERENCES}

1. Linnane BM, Canny G. Congenital broncho-esophageal fistula: a case report. Respir Med 2006;100(10):1855-1857.

2. Azoulay D, Regnard JF, Magdeleinat P, Diamond T, Rojas-Miranda A, Levasseur P. Congenital respiratory-esophageal fistula in the adult: report of nine cases and review of the literature. J Thorac Cardiovasc Surg 1992;104(2):381-384.

3. Braimbridge MV, Keith HI. Oesophago-bronchial fistula in the adult. Thorax 1965;20:226-233.

4. Risher WH, Arensman RM, Ochsner JL. Congenital bronchoesophageal fistula. Ann Thorac Surg 1990;49(3):500-505.

5. Kim JH, Park KH, Sung SW, Rho JR. . Congenital bronchoesophageal fistulas in adult patients. Ann Thorac Surg 1995;60(1):151-155.

6. Aguiló R, Minguella J, Jimeno J, Puig S, Galeras JA, Gayete A, Sánchez-Ortega JM. Congenital bronchoesophageal fistula in an adult woman. J Thorac Cardiovasc Surg 2006;131(4):916-917.

7. Chiu HH, Chen CM, Mo LR, Chao TJ. Gastrointestinal tuberculous bronchoesophageal fistula. J Gastroenterol Hepatol 2006;21(6):1074.

8. Lazopoulos G, Kotoulas C, Lioulias A. Congenital bronchoesophageal fistula in the adult. Eur J Cardiothorac Surg 1999;16(6):667669. 